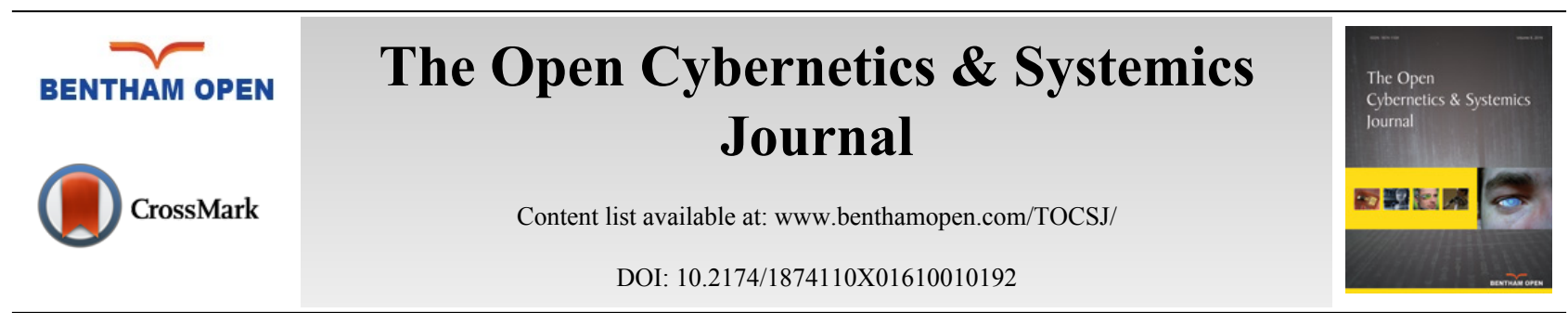

RESEARCH ARTICLE

\title{
A Triangular Cloud Model and Cloud Generator Algorithm
}

\author{
Yanbing Gong*, Liangliang Dai and $\mathrm{Na} \mathrm{Hu}$ \\ Department of Information Management, Hohai University, Changzhou, China
}

Received: October 14, 2015

Revised: August 27, 2016

Accepted: September 05, 2016

\begin{abstract}
Cloud model is a cognitive model which represents the fuzziness and randomness with three digital characteristics, i.e. expectation, entropy and hyper-entropy. The normal cloud model is one of the most important cloud models, which is composed of 2nd-order normal distribution. The normal cloud model is usually expressed in qualitative concept with symmetrical features, and for the concept of fasymmetric only uses the complex combination of cloud model. Therefore, it is more complex in computing and processing in practical application. A new simple cloud model, i.e. triangular cloud model is proposed, and the forward triangular cloud transformation algorithm is presented. Then, a backward triangular cloud transformation algorithm is designed based on the fuzzy discrete expectation. Finally, two simulation examples are given to state the feasibility of the triangular cloud, and the result shows that the new generator algorithm is simple and effective.
\end{abstract}

Keywords: Cloud generator, Fuzzy discrete expectation, Normal cloud model, Triangular cloud model.

\section{INTRODUCTION}

Knowledge representation has been a bottleneck for years in artificial intelligence. The difficulty is uncertainty hidden in qualitative concepts, in particular, randomness and fuzziness [1]. The mathematic methods for handing uncertainty knowledge mostly are probability theory and fuzzy set theory. Probability theory mainly studies the stochastic phenomena, which can deal with stochastic uncertainties well. The normal distribution can be regarded as approximation of a large number of random phenomena [2]. Fuzzy set theory mainly studies the fuzzy phenomena, which mainly use membership degree of the membership function to describe fuzzy events [3]. The Gaussian membership function is considered as one of the most suitable membership function for many fuzzy events. Cloud model was proposed based on probability theory and fuzzy sets theory to describe the uncertainty knowledge in 1995 [4]. The normal cloud model uses three digital characteristics to represent the uncertainty transition between quantitative and qualitative descriptions, which will integrate the randomness and the fuzziness in 2nd-order normal distribution in unified way [5 - 8].

The normal cloud model uses three digital characteristics $(E x, E n, H e)$ to depict the intension of a concept, which just accords with human thought. Expected value Ex is used to show the center position of the normal cloud, whose elements are fully compatible with the uncertainty linguistic concept. The standard variance En is a measure of the granularity scale, which can be used to calculate the membership degree. The hyper-entropy He is a measure of the dispersion on the normal cloud drops, which can also be considered as the entropy of the standard variance En. In order to transform the uncertainty concept of quantitative and qualitative description, Li et al. [1] presented two basic cloud transformation tools, namely forward normal cloud generator (FNCG) and backward normal cloud generator (BNCG). By inputting the number of its characteristics, FNCG produces normal cloud droplets described by the specific concept. BNCG can obtain the qualitative description of $2^{\text {nd }}$-order normal distribution from a series of quantitative cloud droplets which have been given. The two cloud generators can also provide a way to simulate the human bidirectional cognition process for uncertainty concepts by computer program.

\footnotetext{
* Address correspondence to this author at the Department of Information Management, Hohai University, No. 200 North Jinling Road, Changzhou, China; Tel: +865198123 0713; E-mail: yanbg79@163.com
} 
At present, the normal cloud model has been successfully applied in many fields, such as intelligent control [9, 10], data mining [11 - 13], qualitative evaluation [14, 15], image segmentation [16, 17], decision making [18 - 21], and so on. However, the normal cloud model is usually expressed in qualitative concept with symmetrical features, and for the concept of asymmetry only uses the complex combination of cloud model. In fact, a lot of linguistic variables or qualitative concepts are asymmetric in quantification, for example, the concept "Young" in Fig. (2). Normal cloud model needs complex calculation and requires linguistic concept with symmetry in the practical application. Therefore, a simple triangular cloud model is proposed in this paper. The characteristic of triangular cloud model is that the function has the advantage of simple structure, and can unify qualitative representation of the linguistic concept of symmetry and asymmetry, which is conducive to the practical application of uncertain description and problem solving.

The rest of the paper is organized as follows: In Section 2, the concept and generator algorithms of the normal cloud model are introduced. In Section 3, the concepts about triangular cloud model are described. In Section 4, the efficiency of triangular cloud generator algorithms is discussed through two case studies. Finally, Section 5 presents our conclusions and suggestions for future research.

\section{THE NORMAL CLOUD MODEL AND CLOUD GENERATOR}

Normal cloud model is a cognitive method of transformation between quantitative data and qualitative concepts, which can formalize a concept into three numbers. Considering the uncertainty and objectivity of the membership degree, normal cloud model automatically produces the membership degrees based on probability distribution to interpret the fuzziness of concepts, thereby disclosing the relationship between randomness and fuzziness $[1,5]$.

\subsection{The Concept of Normal Cloud Model}

Definition 1 [1]: Let $U$ be the universe of discourse, and $C$ be a qualitative concept in $U$. If $x \in U$ is a random instantiation of concept $C$, which satisfies $x \sim N\left(E x, E n^{2}\right), E n^{\prime} \sim N\left(E n, H e^{2}\right)$, so the degree of certainty of $x$ belonging to concept $C$ satisfies

$$
\mu(x)=e^{-\frac{(x-E x)^{2}}{2 E n^{2}}}
$$

then the distribution of $x$ in the universe $U$ is called a normal cloud or a 2nd-order normal cloud.

The key point in Definition 1 is the $2^{\text {nd }}$-order relationship, i.e. within the two normal random numbers. Specially, if digital characteristic parameter $H e=0$, then the cloud distribution of $x$ on $U$ will become a normal distribution. If digital characteristic parameters $H e=E n=0$, then $x$ will be a constant $E x$ and $\mu(x) \equiv 1$. If digital characteristic parameter $H e$ turns larger, the distribution of random variable $X$ will show a heavier tail, which can be used in economic and social researches.

As shown in Fig. (1), different people have different understanding about the concept of "Young", so it is very different to give a crisp membership degree. However, the normal cloud model can describe this uncertainty concept. Meanwhile, it can also demonstrate the basic certainty of uncertainty.

In normal cloud model, cloud generator, which is a basic tool to realize the transformation between qualitative concepts and quantitative data, is composed of forward cloud generator and backward cloud generator.

\subsection{The Forward Normal Cloud Generator}

The FNCG is the most commonly used in cloud generator, which is a mechanism for mapping form its qualitative representation to its quantitative representation. By inputting the number of its digital characteristics $(E x, E n, H e)$, FNCG produces normal cloud droplets described by the specific concept. The generating algorithm of FNCG (Ex, En, $H e, n)$ can be described as follows $[1,5]$ :

Algorithm 1: Forward normal cloud generator- FNCG $(E x, E n, H e, n)$

Input: Three parameters, Ex, En, and $H e$, and the number of cloud drops $n$

Output: $n$ cloud drops and their certainty degrees

Step 1: A normal random number $E n_{i}^{\prime}$ with expectation of $E n$ and a standard deviation of $H e$ is generated. 


$$
E n_{i}^{\prime}=\text { Normrnd }(\mathrm{En}, \mathrm{He})
$$

Step 2: A normal random number $x_{i}$ with expectation of $E x$ and a standard deviation of $E n_{i}^{\prime}$ is generated.

$$
x_{i}=\operatorname{Normrnd}\left(E x, E n_{i}^{\prime}\right)
$$

Step 3: The certainty degree of $x_{i}$ is calculated as follows:

$$
\mu_{i}=e^{-\frac{\left(x_{i}-E x\right)^{2}}{2 E n_{i}^{\prime 2}}}
$$

Step 4: Then $x_{i}$ is a cloud drop with the confirmation degree $\mu_{i}$ expressed as drop $\left(x_{i}, \mu_{i}\right)$.

Step 5: Repeat the step1 to step4 until the cloud drops generated are sufficient for $n$.

In Fig. (1), this is the result of FNCG $(25,3,0.5,2000)$. It can represent the qualitative concept "Young" or a ratio between 15 and 35 with cloud drops.

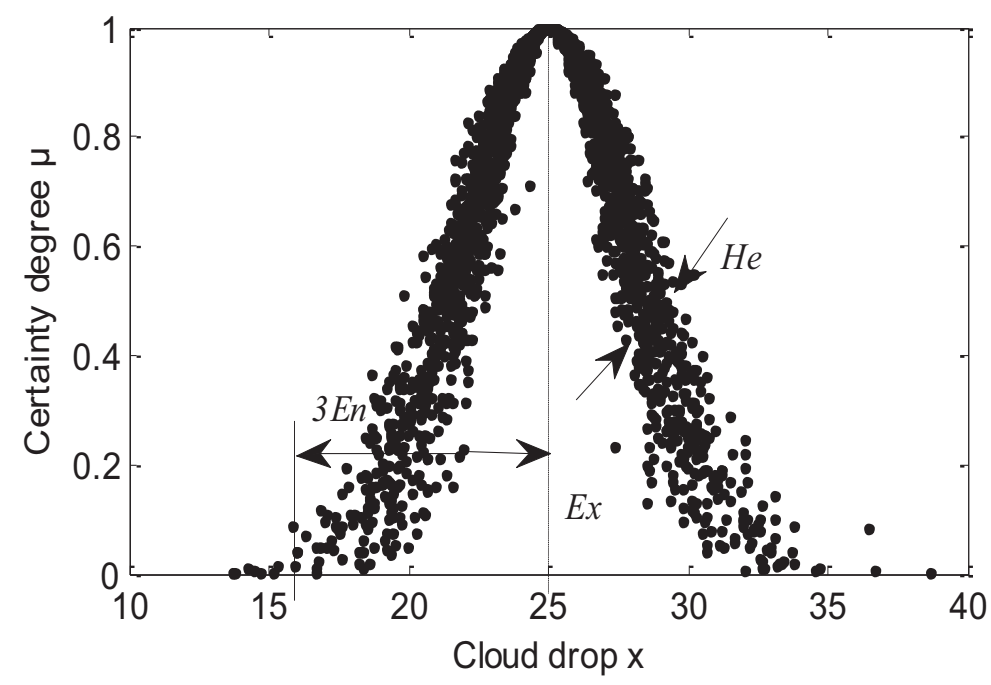

Fig. (1). Describe "Young" by the normal cloud model.

\subsection{The Backward Normal Cloud Generator}

The BNCG is an algorithm based on probability statistics theory. It is used to obtain the quantitative description of three digital characteristic parameters. It generates the cloud parameters $(E x, E n, H e)$ based on the cloud drops $\left(x_{i}, \mu_{i}\right)$. Wang et al. [22] constructed a new algorithm of backward cloud based on the theory of probability statistics, which is more precise than the old. The generating algorithm of the one-dimension normal cloud $\operatorname{BNCG}\left(x_{i}, \mu_{i}\right)$ can be described as follows $[1,5]$ :

Algorithm 2: Backward normal cloud generator- BNCG $(E x, E n, H e, n)$

Input: $n$ cloud drops and their certainty degrees $\left(x_{i}, \mu_{i}\right)$

Output: Three parameters, Ex, En, and $H e$

Step 1: Calculate the mean Ex of cloud drops $x_{i}$

$$
E x=\operatorname{Mean}\left(x_{i}\right)=\frac{1}{n} \sum_{i=1}^{n} x_{i}
$$

Step 2: Calculate the standard deviation En of cloud drops $x_{i}$ 


$$
E n=\operatorname{Stdev}\left(x_{i}\right)=\sqrt{\frac{1}{n} \sum_{i=1}^{n}\left(x_{i}-\bar{X}\right)^{2}}
$$

Step 3: For each cloud drop $\left(x_{i}, \mu_{i}\right)$, calculate the normal random number $E n_{i}^{\prime}$ :

$$
E n_{i}^{\prime}=\sqrt{-\frac{\left(x_{i}-E x\right)^{2}}{2 \ln \left(\mu_{i}\right)}}
$$

Step 4: Then the hyper entropy $H e$ can be calculated by the standard deviation of $E n_{i}^{\prime}$ :

$$
H e=\operatorname{Stdev}\left(E n_{i}^{\prime}\right)
$$

\section{A TRIANGULAR CLOUD MODEL AND CLOUD GENERATOR}

Normal cloud model is an effective tool in uncertain transformation between qualitative concepts and their quantitative expressions. However, compared to the normal cloud model, triangular cloud model has extensive existence in everyday life and its own merits to represent numerical concept. For example, the distribution of the concept "Young" between 15 and 35 is not symmetrical. Obviously, the certainty degree is not the same between interval $(15,25)$ and interval $(25,35)$. For example, 20 years younger than 30 years, so the certainty degree of 20 is larger than 30 . Triangular cloud model can be expressed with symmetry or asymmetry uncertainty linguistic concept.

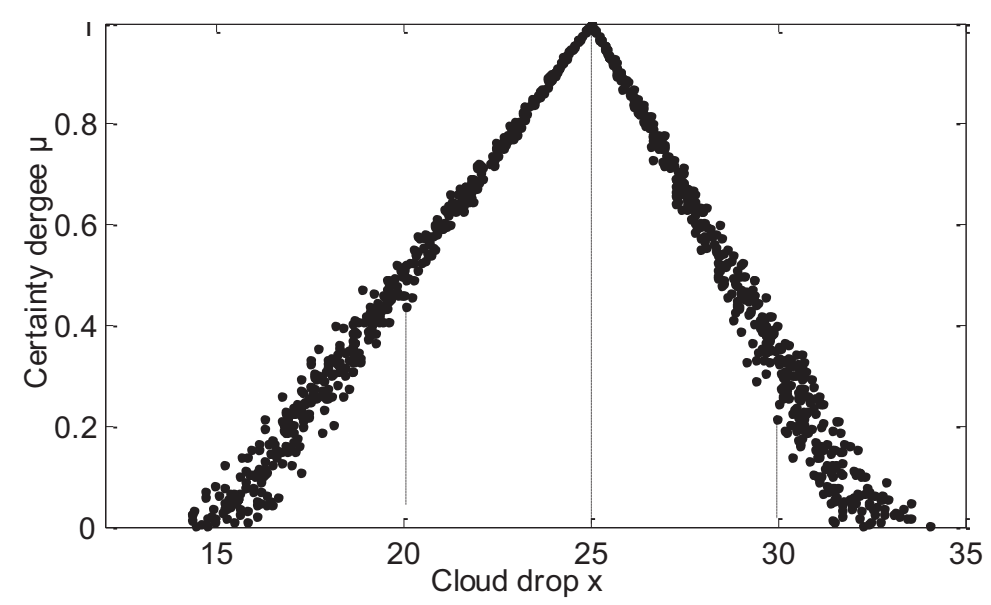

Fig. (2). Describe "Young" by the triangular cloud model.

\subsection{The Concept of Triangular Cloud Model}

Definition 2: Let $U$ be the universe of discourse, and $C$ be a qualitative concept in $U$. If $x \in U$ is a random instantiation of concept $C$, then the degree of certainty of $x$ belonging to concept $C$ satisfies

$$
\mu(x)=\left\{\begin{array}{l}
\frac{x-E a^{\prime}}{E x-E a^{\prime}}, E a^{\prime} \leq x<E x \\
\frac{x-E c^{\prime}}{E x-E c^{\prime}}, E x \leq x \leq E c^{\prime}
\end{array}\right.
$$

where $E a^{\prime} \sim N\left(E a, H e^{2}\right)$ and $E c^{\prime} \sim N\left(E c, H e^{2}\right)$, then the distribution of $x$ in the universe $U$ is called a triangular cloud model.

From the Definition 3.1, triangular cloud model represents the qualitative concept by four digital characteristics( $E a$, $E x, E c, H e)$. The expected value $E x$ is the point which is the most suitable to represent the domain of the concept. The left and right spreads $E a$ and $E c$ are used to figure the granularity scale of the concept. The hyper-entropy $H e$ is used to 
depict the uncertainty of the concept granularity. Moreover, the simple operation rules of triangular cloud model described below make them feasible in practical applications. Specially, if digital characteristic parameter $H e=0$, then the cloud distribution of $x$ on $U$ will become a triangular distribution. The explicit expressions of fuzziness and uncertainty by parameters $(E a, E x, E c, H e)$ make the triangular cloud model easy to understand and compute directly.

Definition 3: Given two triangular clouds $C_{1}$ and $C_{2}$, their digital characters are $C_{1}\left(E a_{1}, E x_{1}, E c_{1}, H e_{1}\right)$ and $C_{2}\left(E a_{2}, E x_{2}, E c_{2}, H e_{2}\right)$, respectively. The arithmetic operation rules of $C_{1}$ and $C_{2}$ can be defined as follows:

$$
\begin{aligned}
& C_{1}+C_{2}=\left(E a_{1}+E a_{2}, E x_{1}+E x_{2}, E c_{1}+E c_{2}, \sqrt{H e_{1}^{2}+H e_{2}^{2}}\right) \\
& C_{1}-C_{2}=\left(E a_{1}-E c_{2}, E x_{1}-E x_{2}, E c_{1}-E a_{2}, \sqrt{H e_{1}^{2}+H e_{2}^{2}}\right) \\
& C_{1} \times C_{2}=\left(E a_{1} \times E a_{2}, E x_{1} \times E x_{2}, E c_{1} \times E c_{2},\left|E x_{1} E x_{2}\right| \times \sqrt{\left(\frac{H e_{1}}{E x_{1}}\right)^{2}+\left(\frac{H e_{2}}{E x_{2}}\right)^{2}}\right. \\
& C_{1} \div C_{2}=\left(E a_{1} \div E c_{2}, E x_{1} \div E x_{2}, E c_{1} \div E a_{2},\left|E x_{1} E x_{2}\right| \times \sqrt{\left(\frac{H e_{1}}{E x_{1}}\right)^{2}+\left(\frac{H e_{2}}{E x_{2}}\right)^{2}}\right)
\end{aligned}
$$

Similar to the normal cloud, the uncertain concept of $C_{1}$ and $C_{2}$ must be in the same universe of discourse such that the triangular cloud operation involved in the algorithm above is meaningful.

\subsection{The Forward Triangular Cloud Generator (FTCG)}

In order to be able to use computer to calculate the triangular cloud model, it is necessary to research the triangular membership cloud generator algorithm. It generates cloud drops based on the cloud parameters $(E a, E x, E c, H e)$. As shown in Fig. (3), the generating algorithm of the one-dimension triangular cloud FTCG $(E a, E x, E c, H e, N)$ can be described as follows:

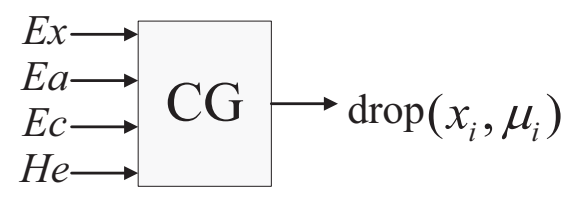

Fig. (3). A forward triangular cloud generator.

Algorithm 3: Forward triangular cloud generator- $\mathrm{FTCG}(E a, E x, E c, H e, n)$

Input: Three parameters, $E a, E x, E c$, and $H e$, and the number of cloud drops $n$

Output: $n$ cloud drops and their certainty degrees

Step 1: Two normal random numbers $E a^{\prime}, E c^{\prime}$ with expectation of $E a, E c$ and a standard deviation of $H e$ is generated.

$$
\begin{aligned}
& E a_{i}^{\prime}=\operatorname{Normrnd}(E a, H e) \\
& E c_{i}^{\prime}=\operatorname{Normrnd}(E c, H e)
\end{aligned}
$$

Step 2: Two uniformly distributed random numbers $x_{1 i}, x_{2 i}$ are generated.

$$
\begin{aligned}
& x_{1 i}=E a_{i}^{\prime}+\left(E x-E a_{i}^{\prime}\right) \times \text { unit } \\
& x_{2 i}=E c_{i}^{\prime}+\left(E x-E c_{i}^{\prime}\right) \times \text { unit }
\end{aligned}
$$

where unit is the random number in interval $[0,1]$.

Step 3: The certainty degree of $x_{i}$ is calculated as follows: 


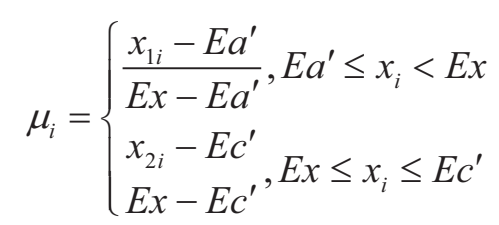

Step 4: Repeat the step1 to step3 until the cloud drops generated are sufficient for $n$.

The algorithm mainly uses a normal random number and a uniform random number. A random number is the foundation of another random number. It is the compound relationship which is the key of the forward triangular cloud generator algorithm. If $H e=0$, the algorithm step 1 always generates a certain value of $E a$ and $E c$, then $x$ has become a triangular membership function. In Fig. (2), this is the simulation result of FTCG $(15,25,32.5,0.5,500)$. It can represent the qualitative language "Young" or a ratio between 15 and 35 with triangular cloud drops.

\subsection{The Backward Triangular Cloud Generator (BTCG)}

The BTCG is an algorithm based on fuzzy set theory. It is used to obtain the quantitative description of four digital characteristic parameters. As shown in Fig. (4), it generates the triangular cloud parameters $(E a, E x, E c, H e)$ based on the cloud drops $\left(x_{i}, \mu_{i}\right)$.

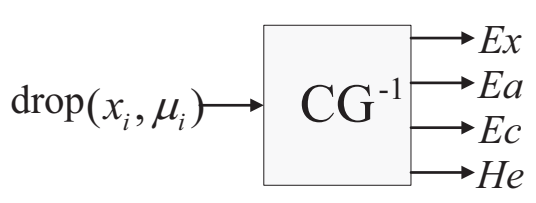

Fig. (4). A backward triangular cloud generator.

Definiton 4 [23]: Let $\xi$ be a normalized fuzzy variable, the expected value of $\xi$ is defined as:

$$
E(\xi)=\int_{0}^{\infty} C r\{\xi \geq r\} d r-\int_{-\infty}^{0} C r\{\xi \leq r\} d r
$$

where $\operatorname{Cr}\{\mathrm{A}\}=0.5(\operatorname{Pos}\{\mathrm{A}\}+\operatorname{Nec}\{\mathrm{A}\})$ is the credibility measure.

Let $\xi$ be a normalized discrete fuzzy variable whose possibility distribution function is defined by:

$$
\mu(x)=\left\{\begin{array}{l}
\mu_{1}, \text { if } x=x_{1} \\
\mu_{2}, \text { if } x=x_{2} \\
\ldots \\
\mu_{n}, \text { if } x=x_{n}
\end{array}\right.
$$

Assume that. It follows form (15) that the expected value of $\xi$ is:

$$
E(\xi)=\sum_{i=1}^{n} x_{i} p_{i}
$$

Then the weights are given by:

$$
p_{i}=\frac{1}{2}\left(\vee_{j=1}^{i} \mu_{j}-\vee_{j=0}^{i-1} \mu_{j}\right)+\frac{1}{2}\left(\underset{j=i}{\vee} \mu_{j}-\underset{j=i+1}{\vee} \mu_{j}\right)
$$

which $\mu=\mu_{\mathrm{n}+1}$ for $i=1,2, \cdots, n$ and satisfy the following constraints:

$$
\sum_{i=1}^{n} p_{i}=\stackrel{n}{\vee} \mu_{i=1}=1
$$


If $\xi$ is triangular fuzzy variable $(E a, E x, E c)$, then the expected value of $\xi$ is:

$$
E(\xi)=\frac{E a+2 E x+E c}{4}
$$

The backward triangular cloud generator algorithm is based on the fuzzy mathematical expectation. The generating algorithm of the one-dimension triangular cloud BTCG $\left(x_{i}, \mu_{i}\right)$ can be described as follows:

Algorithm 4: Backward triangular cloud generator- BTCG $(E a, E x, E c, H e, n)$

Input: $n$ cloud drops and their certainty degrees $\left(x_{i}, \mu_{i}\right)$

Output: Four parameters, Ea,Ex,Ec and He

Step 1: Sort $n$ cloud drops from small to large, i.ex $\leq x_{2} \leq \cdots \leq x_{n}$.

Step 2: Let:

$$
E a=\frac{x_{1}+x_{2}}{2}, E c=\frac{x_{n-1}+x_{n}}{2}
$$

and,

$$
\frac{E a+2 E x+E c}{4}=\sum_{i=1}^{n} x_{i} p_{i}
$$

Then, calculate the three numerical characters $(E a, E x, E c)$. If $\sum_{i=1}^{n} p_{i} \neq 1$, then let $p_{i}=p_{i} / \sum_{i=1}^{n} p_{i}$.

Step 3: For each cloud drop $\left(x_{i}, \mu_{i}\right)$, calculate the normal random number $E a_{i}^{\prime}, E c_{i}^{\prime}$ :

$$
E a_{i}^{\prime}, E c_{i}^{\prime}=\frac{\mu_{i} E x-x_{i}}{\mu_{i}-1}
$$

Step 4: Then the hyper entropy $H e$ can be calculated by the standard deviation of $E a_{i}^{\prime}, E c_{i}^{\prime}$ :

$$
H e=\operatorname{Stdev}\left(E a_{i}^{\prime}\right)=\operatorname{Stdev}\left(E c_{i}^{\prime}\right)
$$

\section{EXPERIMENT ANALYSIS}

Example 1: Let $n=500$, using Matlab programming algorithm can realize the forward triangular cloud generator. For example, let $(E a, E x, E c, H e)=(0.3,0.5,0.8,0.01)$, the forward triangular cloud generator FTCG $(E a, E x, E c, H e, n)$ is shown in Fig. (5).

The source code is as follows:

Input: $(E a, E x, E c, H e)=(0.3,0.5,0.8,0.01)$

$r_{1}=\operatorname{normrnd}(E a, H e,[1500])$;

$r_{2}=\operatorname{normrnd}(E c, H e,[1500])$;

unit=unifrnd $(0,1,[1,500])$;

$x_{1}=r_{1}+\left(E x-r_{1}\right) . *$ unit;

$x_{2}=r_{2}+\left(E x-r_{2}\right) \cdot *$ unit;

output: $\operatorname{plot}\left(x_{1}, 1+\left(x_{1}-E x\right) . /\left(E x-r_{1}\right),{ }^{\prime} .^{\prime}, x_{2}, 1-\left(x_{2}-E x\right) . /\left(r_{2}-E x\right),{ }^{\prime} k{ }^{\prime}\right)$

Example 2: There is a group of cloud drops $\left(x_{i}, \mu_{i}\right)$, and assume that the cloud drops $x_{i}$ have been sorted, as shown in Table 1. 
Table 1. A group of cloud drops.

\begin{tabular}{|l|l|l|l|l|l|l|l|l|}
\hline$x_{i}$ & 0.3276 & 0.3373 & 0.3641 & 0.3735 & 0.3742 & 0.3821 & 0.3950 & 0.3999 \\
\hline$\mu_{i}$ & 0.1359 & 0.1493 & 0.3351 & 0.2853 & 0.3584 & 0.3987 & 0.4374 & 0.6264 \\
\hline$x_{i}$ & 0.4103 & 0.4222 & 0.4490 & 0.4747 & 0.5226 & 0.6148 & 0.6501 & 0.6762 \\
\hline$\mu_{i}$ & 0.5325 & 0.6264 & 0.7258 & 0.8686 & 0.9275 & 0.6226 & 0.5113 & 0.3911 \\
\hline$x_{i}$ & 0.6790 & 0.7086 & 0.7157 & 0.7331 & 0.7514 & 0.7562 & 0.7584 & 0.7666 \\
\hline $\boldsymbol{\mu}_{i}$ & 0.3965 & 0.3100 & 0.2411 & 0.2233 & 0.1326 & 0.1351 & 0.1348 & 0.1102 \\
\hline
\end{tabular}
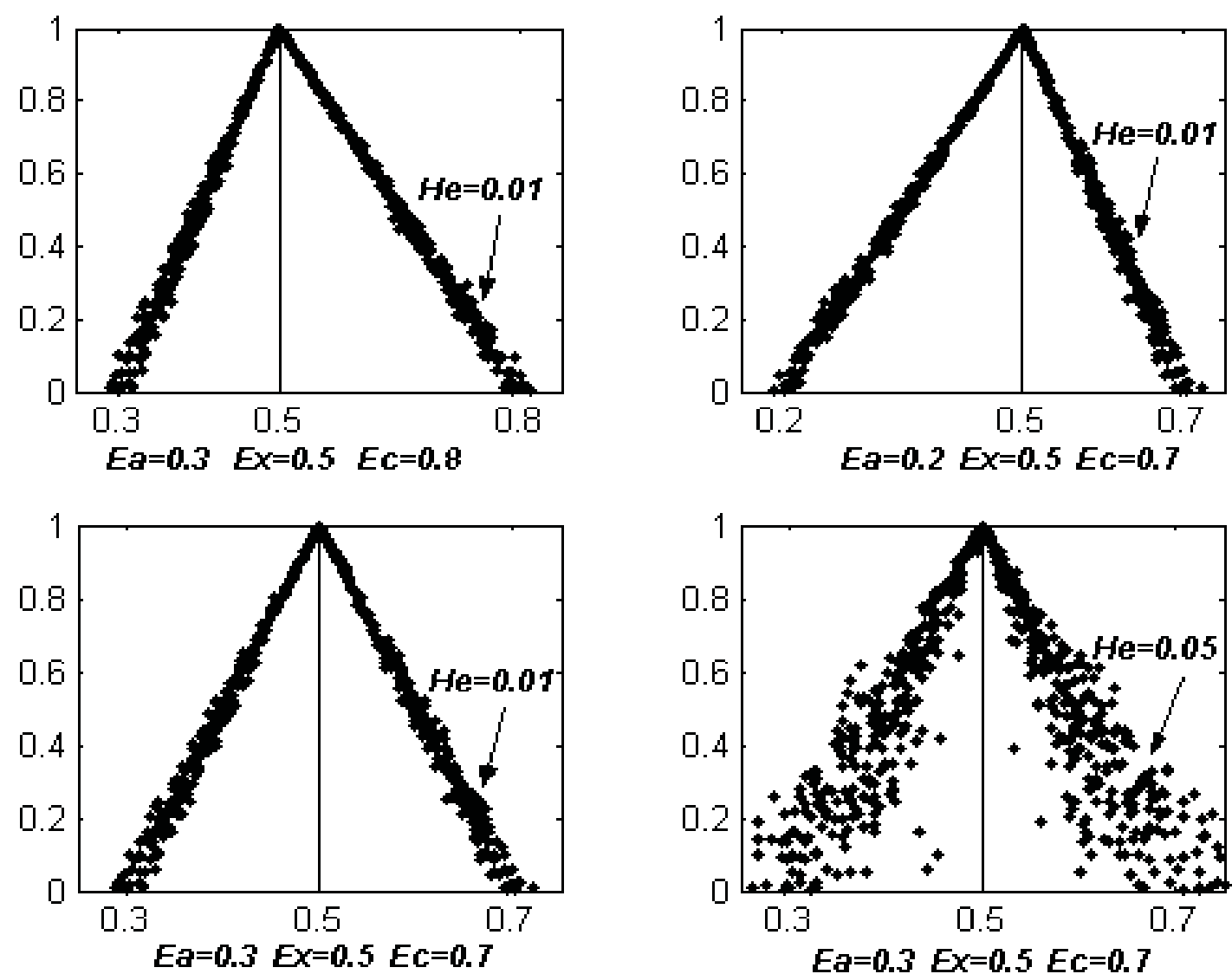

Fig. (5). Different shapes of triangular membership cloud model.

We use the backward triangular cloud generator to calculate the four numerical characters $(E a, E x, E c, H e)$.

With Step 2, we can get $E a=0.3325, E c=0.7625$, and:

$$
p_{1}=\frac{1}{2} \mu_{1}, p_{2}=\frac{1}{2}\left(\mu_{2}-\mu_{1}\right), p_{3}=\frac{1}{2}\left(\mu_{3}-\mu_{2}\right), p_{4}=0, \cdots
$$

If $\sum_{i=1}^{n} p_{i} \neq 1$, let $p_{i}=p_{i} / \sum_{i=1}^{n} p_{i}$, we can get:

$$
\sum_{i=1}^{n} x_{i} p_{i}=0.5205
$$

Then,

$$
\begin{aligned}
& E x \\
& E x=0.4925
\end{aligned}
$$

From Step 3 and step 4, we can get, 


$$
H e=\operatorname{Var}\left(E a_{i}^{\prime}\right)=0.0629
$$

Thus, according to this group of cloud drops, the triangle membership cloud model BTCG $(E a, E x, E c, H e)=\mathrm{BTCG}$ $(0.3325,0.4925,0.7625,0.0629)$, as shown in Fig. (6).

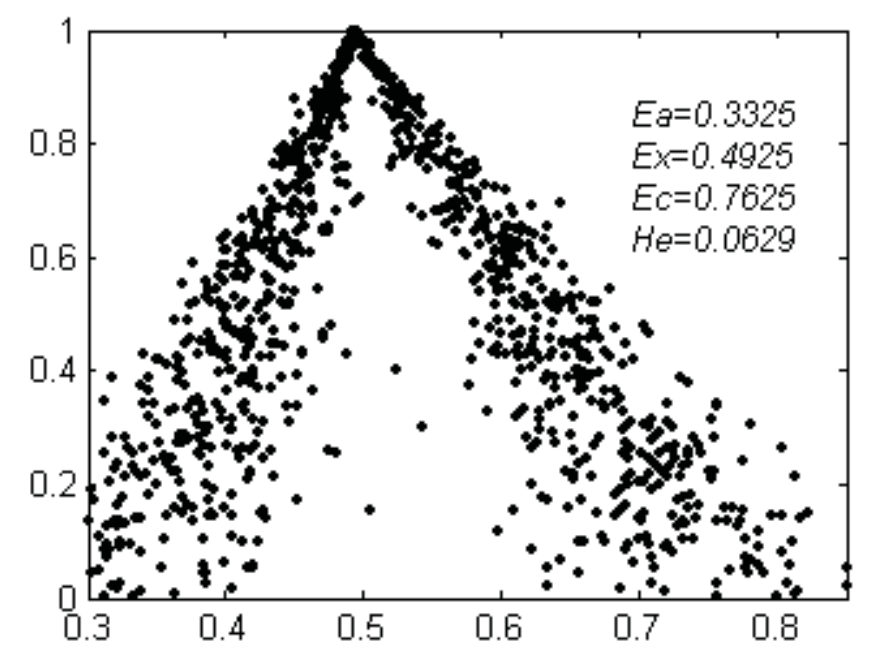

Fig. (6). The backward triangular cloud model.

\section{CONCLUSION}

The cloud model is a good tool for the bidirectional cognitive transformation between the qualitative and quantitative description of a concept by using the cloud generator algorithms. In this paper, based on fuzzy triangular membership function, a simpler and practical cloud model - triangular membership cloud model and the cloud generator algorithms are studied. It has very important significance for the promotion of the application of cloud theory and improves and expands of the existing cloud model theory. Compared with the normal cloud model, the triangular cloud model has two advantages. One is that the triangular cloud model can deal with the symmetric and asymmetric qualitative concepts while the normal cloud model can only deal with the symmetric qualitative concept. Another advantage is that the triangular cloud model is simpler than the normal cloud model in computational process, so it is more convenient for practical application.

In this paper, the backward triangular cloud generator algorithm must be given the value of certainty degree in advance. However, in the practical application, we are often only given a group of cloud drops, whose certainty degree was not given. Error analysis of the backward triangular cloud generator algorithm has not been studied theoretically. Therefore, without the value of certainty degree, the implementation of calculating the four parameters of the triangular cloud and error analysis of the backward generator algorithm will be the focus of our future research work.

\section{CONFLICT OF INTEREST}

The authors confirm that this article content has no conflict of interest.

\section{ACKNOWLEDGEMENTS}

This work was supported in part by the Natural Science Foundation of Jiangsu Province of China (No. BK20130242), the Fundamental Research Funds for the Central Universities (No. 2015B28014), and the Graduate Student Science and Technology Innovation Project of Hohai University Changzhou campus (XZX/ 14B001-07).

\section{REFERENCES}

[1] D.Y. Li, and Y. Du, Artificial Intelligence with Uncertainty., National Defense Industry Press: Beijing, 2014.

[2] Z.K. Wang, Applications of the Probability., Beijing Normal University Press: Beijing, 1995.

[3] L.A. Zadeh, "Fuzzy sets", Inf. Control, vol. 8, pp. 338-353, 1965.

[http://dx.doi.org/10.1016/S0019-9958(65)90241-X] 
[4] D.Y. Li, H.J. Meng, and X.M. Shi, "Membership clouds and membership cloud generators", Int. J. Intell. Syst., vol. 32, pp. 16-21, 1995.

[5] D.Y. Li, J.W. Han, and X.M. Shi, "Knowledge repressentation and discovery based on linguistic atoms", Knowl. Base. Syst., vol. 10, pp. 431-440, 1998. [http://dx.doi.org/10.1016/S0950-7051(98)00038-0]

[6] D.Y. Li, C.Y. Liu, and Y. Du, "Artificial intelligence with uncertainty", J. Softw., vol. 15, pp. 1583-1594, 2004.

[7] D.Y. Li, C.Y. Liu, and W.Y. Gan, "A new cognitive model: cloud model", Int. J. Intell. Syst., vol. 24, pp. 357-375, 2009. [http://dx.doi.org/10.1002/int.20340]

[8] G.Y. Wang, C.L. Xu, and D.Y. Li, "Generic normal cloud model", Inf. Sci., vol. 280, pp. 1-15, 2014. [http://dx.doi.org/10.1016/j.ins.2014.04.051]

[9] F.Z. Zhang, Y.Z. Fan, and C.Z. Shen, "Intelligent control based on membership cloud generators", Chin. J. Electron., vol. 20, pp. 89-92, 1999.

[10] H. Chen, and B. Li, "Approach to uncertain reasoning based on cloud model", J. Chine. Comput. Syst., vol. 32, pp. 2449-2455, 2011.

[11] Y. Liu, D.Y. Li, and G.W. Zhang, "Atomized feature in cloud based evolutionary algorithm", Chin. J. Electron., vol. 37, pp. 1651-1658, 2009.

[12] L.H. Wu, C.L. Zuo, and H.Q. Zhang, "A cloud model based fruit fly optimization algorithm", Knowl. Base. Syst., vol. 89, pp. 603-617, 2015. [http://dx.doi.org/10.1016/j.knosys.2015.09.006]

[13] W.J. Zhang, S.L. Liu, and B. Sun, "A cloud model-based method for the analysis of accelerated life test data", Microelectron. Reliab., vol. 55, pp. 123-128, 2014. [http://dx.doi.org/10.1016/j.microrel.2014.10.006]

[14] H.J. Lu, Y. Wang, and D.Y. Li, "The application of backward cloud in qualitative evaluation", Chine. J. Comp., vol. 26, pp. 1009-1014, 2003.

[15] Y.Z. Shi, and H.C. Zhou, "Research on monthly flow uncertain reasoning model based on cloud theory", Sci. China, vol. 53, pp. 2408-2413, 2010 . [http://dx.doi.org/10.1007/s11431-010-4048-7]

[16] K. Qin, K. Xu, and F.L. Liu, "Image segmentation based on histogram analysis utilizing the cloud model", Comput. Appl. Math., vol. 62, pp. 2824-2833, 2011. [http://dx.doi.org/10.1016/j.camwa.2011.07.048]

[17] T. Wu, and K. Qin, "Image segmentation using cloud model and data field", Pattern Recogn. Artif. Intell., vol. 25 , pp. 397-405, 2012. [http://doi:10.16451/j.cnki.issn1003-6059.2012.03.003].

[18] X.J. Yang, L.L. Yan, and L. Zeng, "How to handle uncertainties in AHP: the cloud delphi hierarchical analysis", Inf. Sci., vol. 222, pp. 384-404, 2013.

[http://dx.doi.org/10.1016/j.ins.2012.08.019]

[19] J.Q. Wang, P. Lu, and H.Y. Zhang, "Method of multi-criteria group decision-making based on cloud aggregation operators with linguistic information", Inf. Sci., vol. 274, pp. 177-191, 2014. [http://dx.doi.org/10.1016/j.ins.2014.02.130]

[20] Z.B. Liu, J.F. Shao, and W.Y. Xu, "Comprehensive stability evaluation of rock slope using the cloud model-based approach", Rock Mech. Rock Eng., vol. 47, pp. 2239-2252, 2013. [http://dx.doi.org/10.1007/s00603-013-0507-3]

[21] D. Wang, D.B. Zeng, and V.P. Singh, "A multidimension cloud model-based approach for water quality assessment", Environ. Res., vol. 149, pp. 113-121, 2016.

[http://dx.doi.org/10.1016/j.envres.2016.05.012]

[22] G.Y. Wang, C.L. Xu, Q.H. Zhang, and X.R. Wang, "A multi-step backward cloud generator algorithm", Springer Berlin Heidelberg: Lecture Notes Comp. Sci, vol. 7413, pp. 313-322, 2012.

[23] B.D. Liu, and Y.K. Liu, "Expected value of fuzzy variable and fuzzy expected value models", IEEE Trans. Fuzzy Syst., vol. 10, pp. 445-450, 2002.

[http://dx.doi.org/10.1109/TFUZZ.2002.800692]

(C) Gong et al.; Licensee Bentham Open

This is an open access article licensed under the terms of the Creative Commons Attribution-Non-Commercial 4.0 International Public License (CC BY-NC 4.0) (https://creativecommons.org/licenses/by-nc/4.0/legalcode), which permits unrestricted, non-commercial use, distribution and reproduction in any medium, provided the work is properly cited. 